\title{
Proton pumps: mechanism of action and applications
}

\author{
Janos K. Lanyi \\ Dept. of Physiology and Biophysics, University of California, Irvine, CA 92697-4560 \\ e-mail jlanyi@orion.oac.uci.edu \\ Andrew Pohorille \\ Exobiology Branch, NASA-Ames Research Center, MS 239-4, Moffett Field, CA 94035 \\ and Dept. of Pharmaceutical Chemistry, University of California San Francisco, CA 94140 \\ e-mail pohorill@max.arc.nasa.gov
}

Recent progress in understanding molecular structures and mechanisms of action of proton pumps has paved the way to their novel applications in biotechnology. Proton pumps, in particular bacteriorhodopsin and ATP synthases, are capable of continuous, renewable conversion of light to chemical, mechanical or electrical energy, which can be used in macro- or nano-scale devices. The capability of protein systems incorporated into liposomes to generate ATP, which can be further used to drive chemical reactions, and to act as molecular motors has been already demonstrated. Other possible applications of such biochemical devices include targeted drug delivery and biocatalytic reactors. All these devices might prove superior to their inorganic alternatives.

In biology, ATP is synthesized at the expense of energy-yielding chemical or photochemical reactions. The ubiquitous ATP synthase (ATPase) takes advantage of transmembrane proton gradients created by the enzymes that catalyze these reactions, with the circulation of protons through these devices providing the means of coupling the exchange of free energy ${ }^{1,2}$. In some organisms it is the ATPase that assumes the role of a pump which, through reversal of its normal function, produces the proton gradient. The membrane proteins that translocate ions, and protons in particular, therefore assume a special place in cellular bioenergetics. How they wbrk, and how the proton gradient they produce is utilized to drive endergonic reactions is an outstahding question of first importance in biology.

The production of desirable reactive chemicals and polymers needs energy input. A human body takes care of its biosynthetic needs by turning over daily an amount of ATP equivalent to approximately its weight (100-200 lbs). It seems unavoidable that the technology of producing biochemicals on an increasingly large scale will have to face the costs of energy input. It will eventually make use of the only truly renewable resource, sunlight and the recycling of a high-energy compound such as ATP. We recount here the great progress in the last few years that promises to reveal the mechanism of proton pumps and the ATPase, and speculate on how biotechnology will make use of such systems.

\section{Proton transport across membranes}

Unassisted transport of protons or, more generally, charges across membranes requires large free energies of activation associated with transfer of these species from the polar, aqueous envi- 
ronment to the nonpolar interior of a membrane. For example, the activation barrier for unassisted transport of $\mathrm{Na}^{+}$was estimated ${ }^{3}$ at $27 \mathrm{kcal} \mathrm{mol}^{-1}$, which means that only a few ions per day could permeate a membrane. For protons another mechanism is also available. It involves translocation of excess protons along hydrogen-bonded chains of water molecules, which, when properly aligned by chance, span the membrane. This mechanism is more efficient because it requires only small displacements of several protons between consecutive water molecules along the chain rather than a large translocation of a single ion. To achieve sustained proton transport an additional step involving the reorientation of water molecules in the chain is required. However, even with the aid of this mechanism the permeability of membranes to protons is quite low. Thus, a membrane forms an excellent permeability barrier to charges, essential for sustaining proton gradients. This is very fortunate because proton leakage is unproductive and is detrimental to the efficiency of bioenergetic processes.

Clearly, to achieve efficient proton transfer across membranes, some assistance is needed. This assistance is provided by transmembrane proteins, which reduce the activation barrier by providing a polar environment to the translocated proton. Again, a proton wire mechanism similar to that postulated for unassisted ion transport is at work. In this case, however, it maight involve not only water molecules, but also protein side-chains. This mechanism, which appears to be universal in membrane biology, has been studied for several transmembrane protein channels. They provide passive pathways for proton transport, i.e., protons are translocated through the channel from the compartment with higher proton concentration to the compartment with lower concentration. In gramicidin $\mathrm{A}$, it has been postulated that protons travel along a single file of water molecules filling the pore of the channel ${ }^{4}$. The situation is somewhat more complicated in the case of a tetrameric channel formed by the M2 protein from human influenza virus. For this channel, an additional step must be involved because the channel contains four histidine amino acid residues, one from each of the helices, which are sufficiently large to occlude the pore and interrupt the water network, thus forming a gate. To explain how protons travel through the gate, it has been proposed that one proton is captured on one side of a histidine residue while another proton is released from the opposite side. Then, the channel returns to the initial state through tautomerization ${ }^{5,6}$.

In contrast to such channels, the so-called Mitchell loops and the pumps are active transport systems, which are able to transport ions also against a concentration and/or electric gradient across the membrane. The loops, as suggested by Peter Mitchell in his landmark chemiosmotic hypothesis $^{7}$, are redox systems inserted asymmetrically into the membrane so as to catalyze the vectorial movement of electrons and accompanying protons between reducing and oxidizihg agents separated by the lipid bilayer. Pumps, on the other hand, contain not only a translocation pathway but also a site of an energy-yielding chemical reaction (bond breakage, isomerization, etc.), and the means of coupling the two together. The loops and the pumps are therefore devices for transducing chemical energy into osmotic and electric potential energy for protons. The resulting protonmotive force is the means for driving energy-requiring reactions via the recirculation of the protons through other devices in the membrane. These other devices are, in effect, loops or pumps that function in reverse. When the energy input (protonmotive force) and energy sink (chemical potential for ATP/ADP, for example) are nearly matched, either one can, in principle, drive the other. In mitochondria, chloroplasts and aerobic bacteria, the balance is toward the ADP + phosphate $\rightarrow$ ATP reaction, making the ATPase device a synthase.

\section{Biological proton pumps}

The long quest towards understanding the mechanism of proton pumps has received a strong boost from high-resolution crystallographic structures determined currently for increasing 
numbers of such proteins. The simplest and best described is bacteriorhodopsin, the retinal-protein of halophilic archaea that functions as a light-driven pump (Fig. 1). Its structure is described to 1.55 $\AA$ resolution ${ }^{8}$, and high-resolution maps have been determined for several intermediates of its transport cycle ${ }^{9.12}$. In this protein, energy input is not from a chemical reaction as in most pumps, but from photoisomerization of the all-trans retinal to 13-cis, which creates a conflict with its binding site and therefore a high-energy state. Relaxation of the retinal and the protein matrix around it causes atomic displacements of protein residues and bound water, and thereby $\mathrm{p} K$ shifts of acidic groups. Most, although not yet all, details of the transport mechanism are now understood. The key event is the protonation of Asp- 85 by the retinal Schiff base (Fig. 1). The consequence of this event is the initiation of a cascade of hydrogen-bond rearrangements that culminate in release of a proton to the extracellular surface. This coupling is mediated by the side-chain of the positively charged Arg82 swinging away from the no longer negatively charged Asp85. Movement of the 13methyl group of the isomerized retinal initiates, in turn, rearrangements in the cytoplasmic region that cause reprotonation of the Schiff base by Asp96, and the uptake of a proton. This is accomplished by displacements of the side-chain of Lys216, where the retinal is attached and the indole ring of Trp 182. The ensuing breaking of hydrogen-bonds and repacking of side-chains causes the outward tilt of one of the transmembrane helices ${ }^{13}$, and allows the intercalation of water in this region, which lowers the $\mathrm{p} K$ of the proton donor asp-96 and opens a proton transfer pathway toward the retinal Schiff base.

Although not yet understood in such detail, it is generally assumed that this kind of coupling between the transformations at the active site and the directional transfer of protons across the membrane will occur in the other pumps. In these pumps, the driving reaction could be electron transfer (in the redox pumps of the mitochondria and photosynthetic reaction centers) ${ }^{14-17}$, transfer of reducing equivalents between $N A D(H)$ and $N A D P(H)$ (in proton-translocating transhydrogenases) ${ }^{18,19}$ or the hydrolysis of ATP (in the membrane ATPases) ${ }^{2}$. The transport mechanisms for such proteins involve complications that arise from coupling to multi-step chemical reaction sequences rather than a single reaction, proton stoichiometries $>1$, and long-distance communication between the driving reaction and the proton translocation that requires large-scale protein motions. The architecture of the ATPase is an example of how some of these problems can be solved ${ }^{1,20}$. This enzyme is a very large supramolecular machine, which contains numerous subunit assemblies of different functions. The larger part, which binds the nucleotide, projects out of the membrane, and the proton translocation is through a rotor with many proton binding sites inside the membrane. The two are connected by a stalk, which contains a stator and a shaft. Rotation of the rotor depends on protons passing through the stator, via an entrance and an exit that opens towdrds the two membrane sides. Its rotation is coupled to sequential conformational changes in the nucleotide binding domain so that one site binds ATP, another ADP and phosphate, and the third is free at any one time during its revolution. The $\mathrm{H}^{+} / \mathrm{ATP}$ stoichiometry is fixed by the number of proton-binding sites on the rotor and the number of nucleotide binding sites on the extra-membrane assembly, and the flexibility of the shaft that transmits the motion of the former to the latter.

\section{Designing synthetic proton pumps}

Do we know enough about proton pumps to design synthetic ones that fulfill specific requirements? Although this goal has not yet been accomplished, it is possible to propose a blueprint of a design that captures the main structural and functional features needed for a simple pump". This blueprint emerges from our understanding of biological proton pumps, and in particular, bacteriorhodopsin and is shown in Fig. 2. A proton source, PS, located near the center of the bilayer initiates the proton transport across the membrane. It could be comprised of a chemical reaction or 
a chromophore and an ionizable species. When protonated, it transfers a proton to the primary proton acceptor A l, which could be, for example, the side-chain of glutamate, aspartate or histidine in the protein. It is required that the acceptor be in close proximity to PS and that the $\mathrm{pK}$ of $\mathrm{Al}$ be coupled to the ionization state of PS. From Al the proton is transferred to the secondary acceptor, A2 and, possibly, further along a hydrogen-bonding chain towards the membrane surface until it is captured by a water molecule and carried to the aqueous environment. The essential requirement in this process is to prevent back transfer of the proton from Al to PS. This can be accomplished if the proton transfer between $A 1$ and $A 2$ is fast and practically irreversible. Several mechanisms, known to exist in proton pumps and channels, are available for this purpose. One of them involves a conformational shift of deprotonated $\mathrm{A} 1$, large enough to disrupt a proton transfer chain. If a transient water molecule bridges A1 with PS or A2, back transfer is impeded by the disruption of this bridge because of the change in the ionization state of $A 1$. If $A 1$ is a histidine residue the mechanism postulated for proton gating in the M2 protein might be in operation and the back reaction is prevented by tautomerization of the imidazole ring.

To maintain the proton gradient, release of the pumped proton must be followed by regeneration of PS using a proton acquired from the opposite side of the membrane. This not only requires appropriate architecture for the proton pump but also depends on the $\mathrm{pH}$ difference between both sides of the membrane. As this difference increases the back reaction becomes progressively more likely and eventually the system approaches a steady state, at which point no further increase of proton gradient is possible.

\section{Proton pumps in ATP-generating systems}

With a natural or an engineered, artificial proton pump reconstituted into a membrane system, together with a proton-translocating ATPase, the generation (or regeneration) of ATP from $\mathrm{ADP}$ and phosphate can be driven by suitable energy input. So far, this type of coupled system has been most effective with light as the source of energy. Both bacteriorhodopsin and the $F_{o}$ portion of $\mathrm{H}^{+}$-ATPases have hydrophobic surfaces and spontaneously insert into lipid bilayers. For less obvious reasons that probably have to do with the electric charge of the two protein surfaces, the orientation of bacteriorhodopsin can be made to be fairly uniform and in the direction of inward pumping. Bacteriorhodopsin is inherently and unusually resistant to both thermal and photo-damage, and if the ATPase is from a thermophile ${ }^{22,23}$, their combination is a sturdy system that is stable and functional for several months or longer. Both proteins can be purified in large amounts (in the case of bacteriorhodopsin, in multi-gram quantities in the laboratory by presently available methods), and by relatively simple biochemical techniques. When proteoliposomes containing both proteins are illuminated, a proton current is generated, inward through bacteriorhodopsin, and outward through the ATPase (Fig. 3), and there is efficient and continuous production of ATP from externally added ADP and phosphate. Although the details of the best conditions for coupling under load conditions need to be worked out, there is sufficient evidence already to suggest that this system might be useful for biotechnological applications when ATP regeneration is required. Another established way to drive ATP synthesis is with a synthetic system based on the idea of a Mitchell loop. It uses not a proton pump but a redox chain consisting of linked carotene-porphyrinnaphtoquinone that spans the liposome membrane ${ }^{24}$. Light causes the migration of an electron, oxidizing the carotene moiety and reducing the naphtoquinone, on the interior and exterior surfaces, respectively. A mobile quinone returns the dislocated electron and a proton with it, to the interior surface, generating thereby an inward-directed electrochemical proton gradient, in a reaction cycle that resembles those of proton pumps.

Potential biological applications and perspectives 
Proton pumps, alone or coupled with ATP-generating ATPases, might have numerous biotechnological applications, ranging from targeted drug delivery to biocatalytic reactors, fuel cells and nano-machines. However, the technological potential of proton pumps has been, so far, poorly explored. Recent advancements in our understanding of the structure and mechanism of action of these pumps, combined with rapid progress in several other areas of biotechnology, such as novel application of liposomes or fabrication of biocompatible namostructures, open for the first time a possibility to realize fully this potential.

One potential application of proton pumps is in $\mathrm{pH}$-sensitive liposome systems used for drug delivery, vaccination, gene therapy and medical diagnostics ${ }^{25.26}$. These liposomes are stable at neutral $\mathrm{pH}$ but become destabilized and release their content upon acidification. If the liposome membrane contained a light-driven proton pump, for example bacteriorhodopsin, the release could be triggered by tissue or skin illumination. This technique could be particularly efficient if wavelengths to which biological tissue is relatively transparent were used. The absorption maximum of bacteriorhodopsin is at $570 \mathrm{~nm}$, but it absorbs light between about 500 and $630 \mathrm{~nm}$. This range can be extended by replacing the retinal with various chemical analogues. Thus, wavelengths can be used that are weakly absorbed by water and hemoglobin. By exerting spatial and temporal control of illumination it should be possible to increase markedly the chances that the liposome content is released at the desired target, which might significantly reduce toxic side effects and increase the efficacy of the treatment. More generally, proton pumps might provide the means to use light and accompanied changes of $\mathrm{pH}$ to regulate precisely molecular assemblies and biochemical reactions.

Proton pumps coupled with ATPases provide the means to covert light into chemical energy in a continuous and renewable manner, and further utilize this energy in a variety of biotechnological applications. One such application might be biocatalytic membrane reactors ${ }^{27}$. In these reactors, enzymes suspended in solution inside a membrane-surrounded compartment, or immobilized within the membrane, catalyze the desired chemical reactions. This form of biocatalysis is used in agro-food, detergent, textile, chemical and pharmaceutical industries. However, current applications are limited to enzymatic reactions that do not require the input from high-energy compounds, such as ATP. The range of applicability of biocatalytic reactors can be greatly extended by adding proteoliposomes containing bacteriorhodopsin and ATPase to the membrane-enclosed compartment. Then, upon illumination and in the presence of ADP and inorganic phosphate in the compartment the reactor will support catalysis of ATP-driven reactions. To make this approach technologically viable, the reported rate of continuous ATP production at saturating light intensity of 0.2-0.8 nmol of ATPmin ${ }^{-1} \mathrm{mg}^{-1}$ of protein might have to be improved. This can be achieved, for example, by optimizing conditions for insertion of the proteins into liposomes in the correct orientation and improving efficiency of coupling between the protein components.

ATP generated by ATPases coupled to proton pumps can be used to improve performance of microfabricated biocatalytic fuel cells ${ }^{28}$. These devices can provide electrical power supply for implantable microsurgery robots, biosensors or even artificial organs. One particularly attractive type of fuel cell is the enzyme battery, in which the oxidation and reduction reactions are catalyzed by properly chosen enzymes, immobilized on the electrodes. In such a device, light-driven, ATPproducing proteoliposomes can be used to regenerate the battery. In this respect the system would resemble batteries used, for example, in pocket calculators that recharge when exposed to light.

An appealing application of proton pumps and ATPases is as molecular motors. ATPase contains two rotary motors. Depending on whether the chemical potential of ATP/ADP or the protonmotive force is greater, either one or the other dominates. The $F_{1}$ motor generates a mechanical torque during the hydrolysis of ATP. The $F_{0}$ motor generates a torque in the opposite direction 
when employing the transmembrane protonmotive force. A remarkable feature of the $F_{1}$ motor is that at high ATP concentrations it can convert energy from hydrolysis of ATP to rotary torque at nearly $100 \%$ efficiency ${ }^{20,29}$. The force generated by this motor exceeds $100 \mathrm{pN}$, which is the greatest among molecular motors. Recently, $F_{1}$ ATPase was successfully attached to nickel, copper and gold substrates using electron beam litography ${ }^{30}$. Fluorescent microspheres attached to the tip of the rotating subunit were shown to move with rotational velocity of 3-4 rev s${ }^{-1}$. Continuing work along these lines might lead to the construction of new, autonomous nanomechanical devices powered by proton pumps coupled to ATPases.

It should be clear from the foregoing that although the biophysics of these molecular devices is well understood, their utilization in working systems needs new and creative thinking in micro-engineering. The scale on which they are to be constructed, and on which they will operate, is vastly smaller than the dimensions of state-of-the-art electrical microcircuits, present and projected. The concepts, and in some cases the beginnings of working laboratory models, have been developed. The barrier to overcome is the engineering of reproducible biochemical devices that show clear superiority in reliability and cost to their inorganic alternatives. Whether in biotechnology, nano-scale medical devices, or molecular electronics, the opportunities and the challenges are unprecedented. How rapidly they will be realized depends on the vision that drives the development of industry in the $21^{\text {st }}$ century.

\section{Conclusions}

Proteoliposomes containing proton pumps and ATPases are probably the most promising, general-purpose biodevices for efficient, continuous and renewable generation of energy in biotechnology, especially at the nano-scale. These systems are sturdy and can be produced at technological scales. Other potential applications of proton pumps rely on their ability to change acidity of the environment in a controlled way and, by doing so, influence molecular structures and chemical reactions. With the emerging understanding of how proton pumps work, it is possible to re-engineer them to optimize their usefulness in specific applications. However, the challenges that have to be met in the development of commercial applications of proton pumps are not yet fully known, and considerable work is needed before devices containing pumps become common in bio- and nanotechnology.

\section{References}

1.Abrahams, J.P. et al. (1994) Structure at 2.8 A resolution of $F_{1}$-ATPase from bpvine heart mitochondria Nature 370, 621-628

2.Boyer P.D. (2000) Catalytic site forms and controls in ATP synthase catalysis. Biochim Biophys. Acta $1458,252-62$

3.Wilson, M.A. and Pohorille, A. (1996) Mechanism of unassisted ion transport across membrane bilayers. J. Am. Chem. Soc., 118, 6580-6587

4.Akeson, M. and Deamer, D.W. (1990) Proton conductance by the gramicidin water wire. Model for proton conductance in the $\mathrm{F}_{0} \mathrm{~F}_{1}$ ATPases? Biophys. J. 60, 101-109

5.Pinto, L.H. et al. (1997) A functionally defined model for the M2 proton channel of Influenza A virus suggests a mechanism for its ion selectivity. Proc. Natl. Acad. Sci. U.S.A. 94, 11301-11306 
6.Schweighofer, K.J. and Pohorille, A. (2000) Computer simulation of ion channel gating: The M2 channel of Influenza A virus in a lipid bilayer. Biophys.J. 78, 150-163

7.Mitchell, P. (1966) Chemiosmotic coupling in oxidative and photosynthetic phosphorylation. Research report no. 66/1, Glynn Research, Bodmin, England

8.Luecke, H. et al. (1999) Structure of bacteriorhodopsin at 1.55 Angstrom resolution. J. Mol. Biol. $291,899-911$

9.Edman, K. et al. (1999) High-resolution x-ray structure of an early intermediate in the bacteriorhodopsin photocycle. Nature 401, 822-826

10.Luecke, H. et al. (1999) Structural changes in bacteriorhodopsin during ion transport at 2 angstrom resolution Science 286. 255-261

11.Luecke, H. et al. (2000) Coupling photoisomerization of the retinal in bacteriorhodopsin to directional transport. J. Mol. Biol. 300, 1237-1255

12.Sass, H.J. et al. (2000) Structural alterations for proton translocation in the $M$ state of wild-type bacteriorhodopsin. Nature 406, 649-653

13.Subramaniam, S. and Henderson, R. (2000) Molecular mechanism of vectorial proton translocation by bacteriorhodopsin. Nature 406, 653-657

14.Iwata, S. et al. (1995) Structure at $2.8 \AA$ resolution of cytochrome c oxidase from Paracoccus denitrificans. Nature $376,660-669$

15.Iwata, S. et al. (1998) Complete structure of the 11 -subunit bovine mitochondrial cytochrome Bcl complex. Science 281, 64-71

16.Tsukihara, T. et al. (1996) The whole structure of the 13-subunit oxidized cytochrome c oxidase at $2.8 \AA$. Science $272,1136-1144$

17.Deisenhofer, J. and Michel, H. (1991) High-resolution structures of photosynthetic reaction centers. Annu. Rev. Biophys. Biophys. Chem. 20, 247-266

18.Jackson, B. J. et al. (1999) Structure and mechanism of proton-translocating transhydrogenase. FEBS Lett. 464, 1-8

19.Bizouarn, T. et al. (2000) The transmembrane domain and the proton channel in protonpumping transhydrogenases.. Biochim. Biophys. Acta 1459, 284-290

20.Elston, T. et al. (1998) Energy transduction in ATP synthase. Nature 391, 510-513 
21.Pohorille, A. et al. (1996) Molecular modeling of protocellular functions, in Pacific Symposium on Biocomputing, (Hunter, L. and Klein, T.E., eds.), pp. 550-569, World Scientific, Singapore

22.Pitard, B. et al. (1996) ATP synthesis by the $\mathrm{F}_{0} \mathrm{~F}_{1}$ ATP synthase from thermophilic Bacillus PS3 reconstituted into liposomes with bacteriorhodopsin. 1. Factors defining the optimal reconstitution of ATP synthases with bacteriorhodopsin. Eur. J. Biochem. 235, 769-778

23.Pitard, B. et al. (1996) ATP synthesis by the $\mathrm{F}_{0} \mathrm{~F}_{1}$ ATP synthase from thermophilic Bacillus PS3 reconstituted into liposomes with bacteriorhodopsin. 2. Relationships between proton motive force and ATP synthesis. Eur. J. Biochem. 235, 779-788

24.Steinberg-Y frach, G. et al. (1998) Light-driven synthesis of ATP catalysed by $F_{0} F_{1}-A T P$ synthase in an artificial photosynthetic membrane. Nature 392, 479-482.

25.Chu, C.J. et al. (1990) Efficiency of cytoplasmic delivery by pH-sensitive liposomes to cells in culture. Pharm. Res. 7, 824-834

26.Zignani, M. et al. (2000) In vitro characterization of novel polymeric-based $\mathrm{pH}$-sensitive loposome systems. Biochim. Biophys. Acta 1463, 383-394

27.Giorno, L. and Drioli, E. (2000) Biocatalytic membrane reactors: applications and perspectives. Trends Biotechnol. 18, 339-349

28.Sasaki, S. and Karube, I. (1999) The development of microfabricated biocatalytic fuel cells. Trends Biotechnol. 17, 50-52

29. Noji, H. et al. (1997) Direct observation of the rotation of $F_{1}$-ATPase. Nature 386, 299-302

30.Montemagno, C. and Bachand, G. (1999) Constructing biological motor powered nanomechanical devices. Nanotechnol. 10, 225-231

\section{Figure captions}

Fig. 1. Crystallographic structure of bacteriorhodopsin ${ }^{8}$ (PDB code $1 C 3 \mathrm{~W}$ ). The seven transmembrane helices span the membrane, shown with the cytoplasmic surface facing upward. The amino acids most relevant for proton transport as discussed in the text, the all-trans retinal (in purple), and water molecules (in green) are included.

Fig. 2. Schematic of a simple, artificial, light-driven proton pump. Upon illumination, the proton source PS donates proton to the acceptor Al. The proton is further irreversibly carried to the aqueous environment with the aid of other proton acceptors in the protein and, possibly, water molecules (as shown in this figure). The proton source is reprotonated from the opposite side of the membrane. 
Fig. 3. Schematic of a coupled bacteriorhodopsin - ATP synthase proteoliposome. Proton circulation is maintained during illumination by inward active transport through bacteriorhodopsin, and passive outward flux through the $F_{0}$ part of the ATP synthase. 


$$
\frac{35}{3}
$$




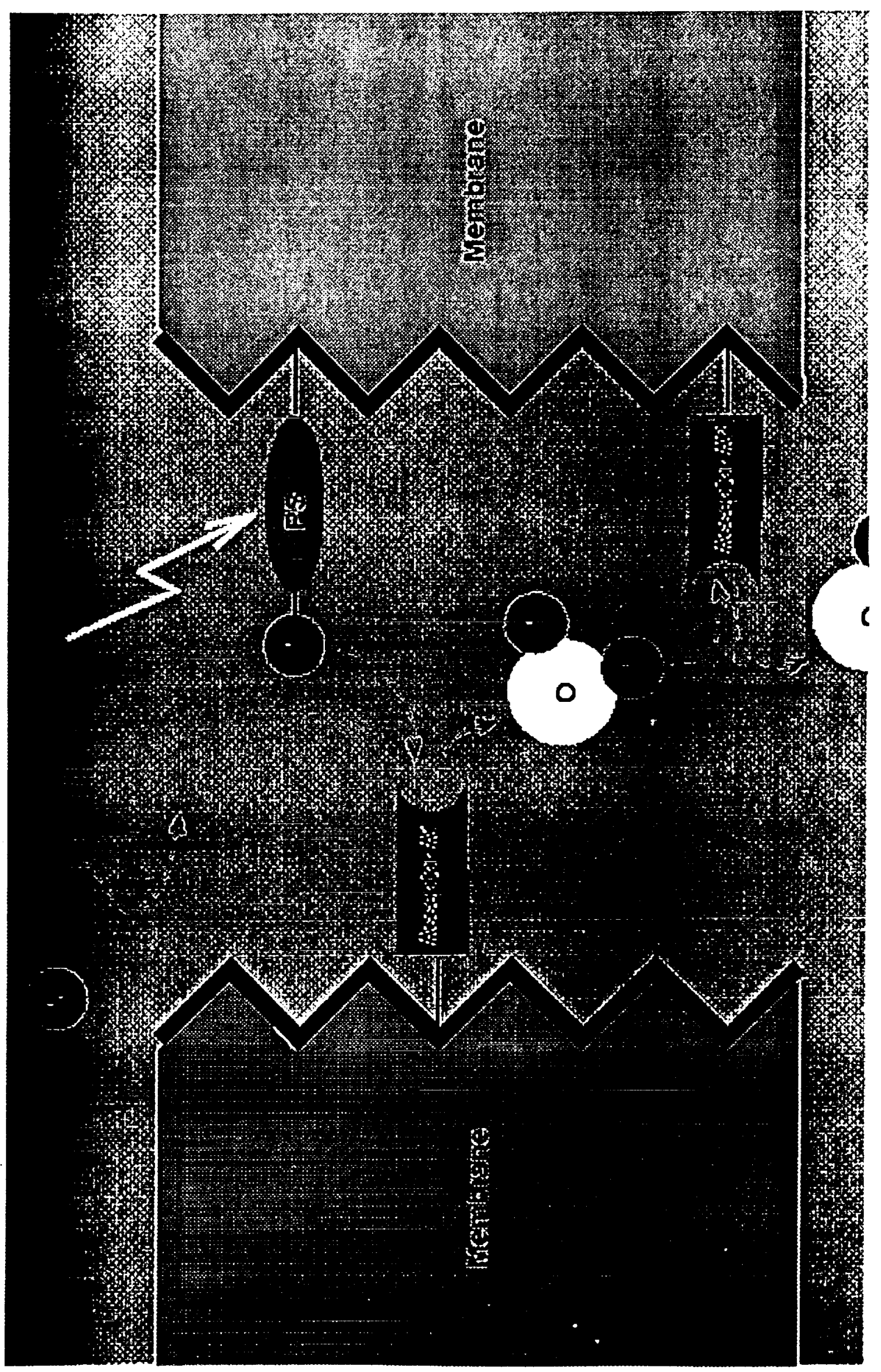




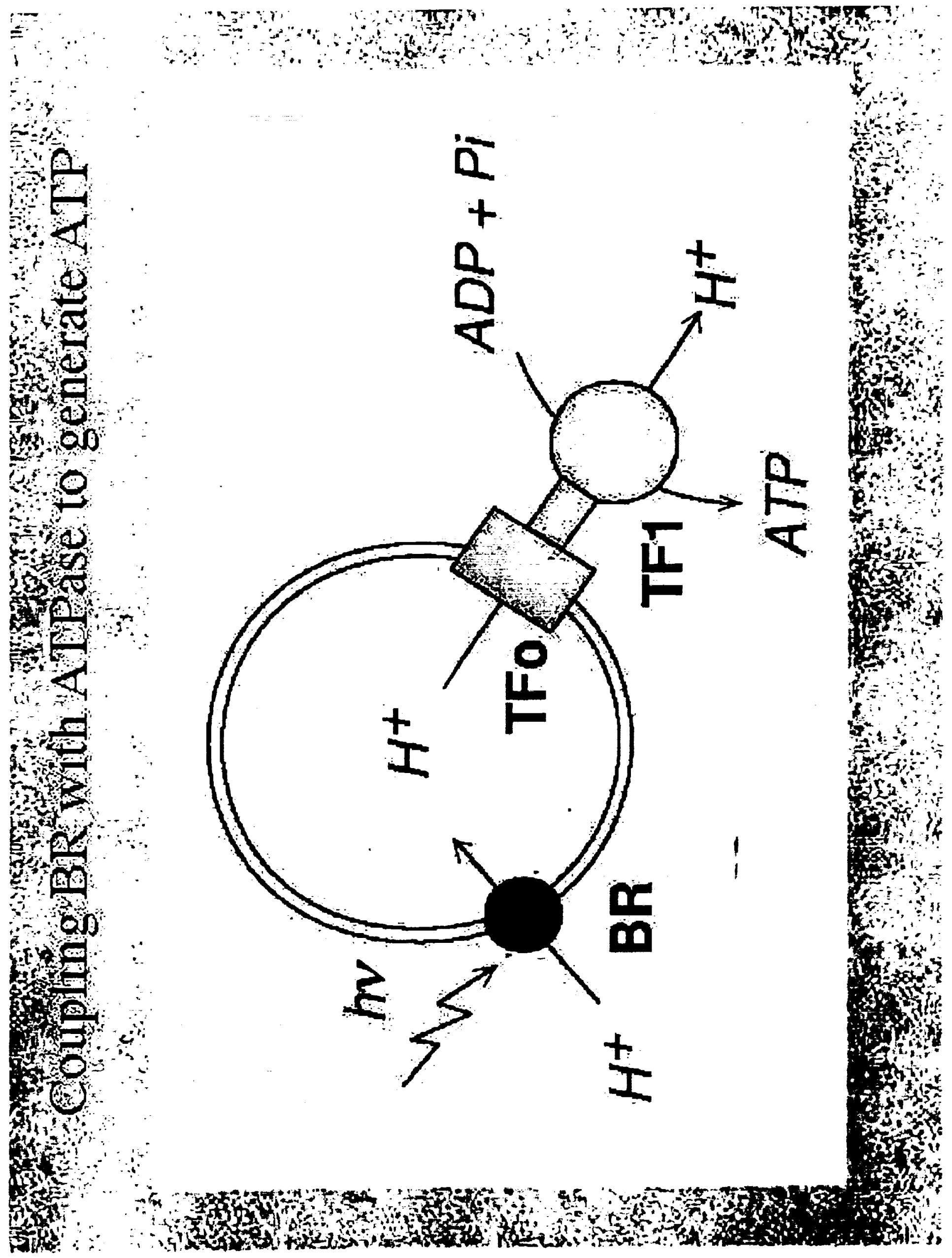

